\title{
Modeling for metabonomic fingerprint assignment in olive fruits
}

\author{
N. Hernández-Sánchez and P. Barreiro \\ Physical Properties Laboratory-Advanced \\ Technologies in Agri-food (LPF-TAG), \\ Madrid, Spain
}

\section{J. Ruiz-Cabello}

Instituto de Estudios Biofuncionales (IEB), Madrid, Spain

\author{
L. León \\ Instituto Andaluz de Investigación y \\ Formación Agraria, Pesquera, Alimentaria \\ y de la Producción Ecológica (IFAPA), \\ Córdoba, Spain
}

Keywords: metabonomics, metabolic fingerprinting, olive, internal quality

\begin{abstract}
Metabonomics has been recently applied to a variety of studies in the agrifood field, maily focused on adulteration identificacion and cultivar or geographical origin assessment. One-dimensional HR-NMR spectrum was acquired using a standard 1D pulse sequence (NOESYPRESAT) with water peak suppression. Twodimension correlation spectroscopy analyses, ${ }^{1} \mathrm{H}-{ }^{1} \mathrm{H}$ 2D experiments (COSY90), were performed. ACD/LABS 8.00 software package was used to obtain simulated spectra from main components in olive, and minor components including those responsible for antioxidant characteristic and aromatics. The application of multivariate statistical techniques to a large HR-NMR spectra data base is the next step.
\end{abstract}

\section{INTRODUCTION}

NMR-based chemometrics is an analytical technology based on spectroscopy in combination with mathematical modeling. The high resolution (HR) Nuclear Magnetic Resonance (NMR) spectrum of a biological tissue provides its $n$-dimensional metabolic fingerprint, whose dimensions could be simply the spectral intensity distribution or the concentrations of individual measurable metabolites [1]. When the complex data sets obtained with NMR spectroscopy were first interpreted using multivariate analysis the concept of Metabonomics was born (Lindon et al., 2006). Metabonomics has been recently applied to a variety of studies in the agri-food field, maily focused on adulteration identificacion and cultivar or geographical origin assessment.

Spain is the most important producer of olive oil in the world and therefore accurate and reliable analitical techniques are key tools for the characterization of quality, mainly as fat content, which is currently determined by means of low-resolution Nuclear Magnetic Resonance (LR-NMR) spectroscopy. High-resolution NMR (HR-NMR) spectroscopy allows the simultaneous analysis of a variety of chemical components. Within the last few years, the development of a technique called high-resolution magic angle spinning (MAS) NMR spectroscopy has made feasible the analysis of small pieces of intact tissues with no pretreatment. The solid or semi-solid sample is placed in a rotor device for rapid spinning (tipically at $\sim 4-6 \mathrm{kHz}$ ) at an angle of $54.7^{\circ}$ relative to the applied magnetic field so as to average out the magnetic field inhomegeneities caused by the sample itself and thus, achieving fine resolved peaks. Two-dimension correlation spectroscopy analyses and programs for spectra simulation from known molecules are 
implemented as assistance for identification of the metabolites responsible for the spectral lines appearing in the one-dimension HR-NMR spectra.

The aim of this work is to establish the starting point to characterize cultivars in the breeding program of the "Instituto Andaluz de Investigación y Formación Agraria, Pesquera, Alimentaria y de la Producción Ecológica" (IFAPA) through the metabolite riddle hidden within the metabonomic fingerprinting of olive flesh.

\section{MATERIALS AND METHODS}

Olea europaea L. fruits were obtained from three different parentals (cv. Picual, cv. Arbequina and cv. Frantoio) and six descendants (Table 1). For each sample highresolution (Bruker AMX-500 spectrometer) ${ }^{1} \mathrm{H}$ NMR spectra were obtained at LPF-TAG.

Pieces of flesh $(2 \times 2 \times 2 \mathrm{~mm})$ were extracted from olive fruits. These samples were packed into a $4 \mathrm{~mm}$ HR-MAS zirconium oxide rotor with $50 \mu \mathrm{l}$ cylindrical insert and spun at $4000 \mathrm{~Hz}$ spinning rate (Fig.1).

One-dimensional HR-NMR spectrum was acquired using a standard 1D pulse sequence (NOESYPRESAT) with water peak suppression. The position of each spectral band is known as chemical shift. It is measured in frequecy terms, in parts per million, from the position of the added reference substance 3-trimethylsilylpropionic acid (TSP). Spectra were reduced into consecutive integrated spectral regions of $0.04 \mathrm{ppm}$ width and individually normalized with respect to the total sum of the whole spectrum. Such resolution $(0.04 \mathrm{ppm})$ was selected according to reviewed literature (Lindon et al., 2001) for avoidance of $\mathrm{pH}$ efect on chemical shift. Two-dimension correlation spectroscopy analyses, ${ }^{1} \mathrm{H}-{ }^{1} \mathrm{H} 2 \mathrm{D}$ experiments (COSY90), were performed. Samples were maintained at $4^{\circ} \mathrm{C}$ in order to precent from degradation.

ACD/LABS 8.00 software package was used to obtain simulated spectra from main components in olive: oleic acid, palmitic acid, linoleic acid, stearic acid and palmitoleic acid; and minor components including those responsible for antioxidant characteristic and aromatics: squalene, tyrosol, benzoic acid, hexanol and metal-butanol among others (Fig.3 and Fig.4).

Analysis of variance (ANOVA) was performed between groups of olives in order to determine the spectral variables, that is, the chemical shifts with significant differences in the corresponding signal intensity. Those chemical shifts were compared to simulated spectra as a first attempt of identifying the component giving rise to the signal and hence, the differences between parentals and descendants.

\section{RESULTS}

One-dimensional ${ }^{1} \mathrm{H}$ HR-NMR spectra showed a great number of peaks at different chemical shifts (Fig.2). Nuclei of hydrogen atoms in different chemical environment appear at different positions within the spectrum. As the strong water signal was suppressed during data acquisition the dominant peaks primary correspond to hydrogens within fatty acids since these molecules are the most abundant components. The intensities (integrals) are proportional to the number of protons present in each functional group.

The dominant signal intensities appear around 0.92, 1.31, 1.57, 2.04, 2.27, and $5.35 \mathrm{ppm}$ chemical shifts (Fig.2). Acording to simulated spectra and to reviewed literature those peaks are asociated with different fatty acids (Fig.3). Only a few of this peaks showed significant differences between groups. Most of the chemical shifts with different signal intensities lie within the region between 3 and 5 ppm (Table 2), also being critical 
signals around 9 and $10 \mathrm{ppm}$. These regions include the chemical shifts for phenolic compounds such as verbascoside and demethyloleuropein (Servili et al., 1999) and triglycerides and diglycerides as well as different aldehydes (Sacchi et al., 1996).

Two-dimensional HR-NMR spectra (Fig.5) show increasing signal dispersion and elucidate the conectivities between signals providing information on which hydrogens in a molecule are close in terms of chemical bonds, which is a valuable clue for peak assignment.

\section{DISCUSSION}

The interest in Metabonomics arises from the feasibility of monitoring complex structures and of performing qualitative and quantitative analysis for structural and compositional studies. In comparison to other analytical techniques Metabonomics provides simultaneous information on a variety of compounds with minimum sample preparation.

In fact, Mass spectrometry (MS) has also been widely used in metabolic fingerprinting and metabolite identification (Lindon et al., 2006). This technique is more sensitive than NMR spectroscpy. However, some drawbacks are remarkable such as the requirement of prior separation of the complex mixture sample using cromatography. Different extraction schemes and separation techniques are applied for different classes of substances and procedures are often cumbersome. Thus, a variety of chromatograms are obtained for each class of substances with extensive total running time.

In contrast, high resolution NMR spectra are a source of information that provides huge amount of data in a single experiment where all the components in the sample are detected independently of their different nature. Nevertheless, it must be highlighted that both techniques are highly complementary for full molecular characterization as stated by Lindon et al. (2006).

The application of multivariate statistical techniques to the HR-NMR spectra would allow the metabonomic fingerprinting of any sample or class so that reliable characterization and classification is feasible with the certainty of every component is taking part in the deal.

\section{CONCLUSIONS}

Metabonomics provides a valuable and reliable tool for characterizing and classifying cultivars in a breeding program. Next step is to obtain a large data base from spectra obtained from a high number of samples so that multivariate statistical techniques can be applied obtainig results with statistical significance.

\section{ACKNOWLEDGEMENTS}

We gratefully acknowledge David Castejón ad Palmira Villa from CAIResonancia Magnética Nuclear for technical assistance, and TAGRALIA-CM and IMDEA for financing this work.

\section{Literature Cited}

Guillén M.D., Ruiz A. High resolution 1H nuclear magnetic resonance in the study of edible oils and fats. Trends in Food Science \& Technology 2001, 12, 328-338.

Lindon J.C., Holmes E., Nicholson J.K. Metabonomics Techniques and Applications to Pharmaceutical Research \& Development. Pharm.Res. 2006, 23 (6), 1075-1088. 
Nicholson, J.K., Lindon, J.C., Holmes, E. "Metabonomics": understanding the metabolic responses of living systems to pathophysiological stimuli via multivariate statistical analysis of biological NMR spectroscopic data. Xenobiotica, 1999, 29 (11): 11811189.

Servili M, Baldioli M., Selvaggini R, Miniati E, Macchioni A, Montedoro G, 1999. Highperformance liquid chromatography evaluation of phenols in olive fruit, virgin olive oil, vegetation waters, and pomace and 1D- and 2D-nuclear magnetic resonance characterization. J. Am. Oil Chem. Soc. 76 (7): 873-882.

Sacchi, R, Batumi, M., Fontanazza, G., Barone, P., Fiordiponti, P., Mannina, L., Rossi, E. and Segre, A.L. 1996. A high-field ${ }^{1} \mathrm{H}$ Nueclear Magnetic Resonance study of the minor components in virgin olive oils. JAOCS, 73:747-758.

\section{$\underline{\text { Figures }}$}
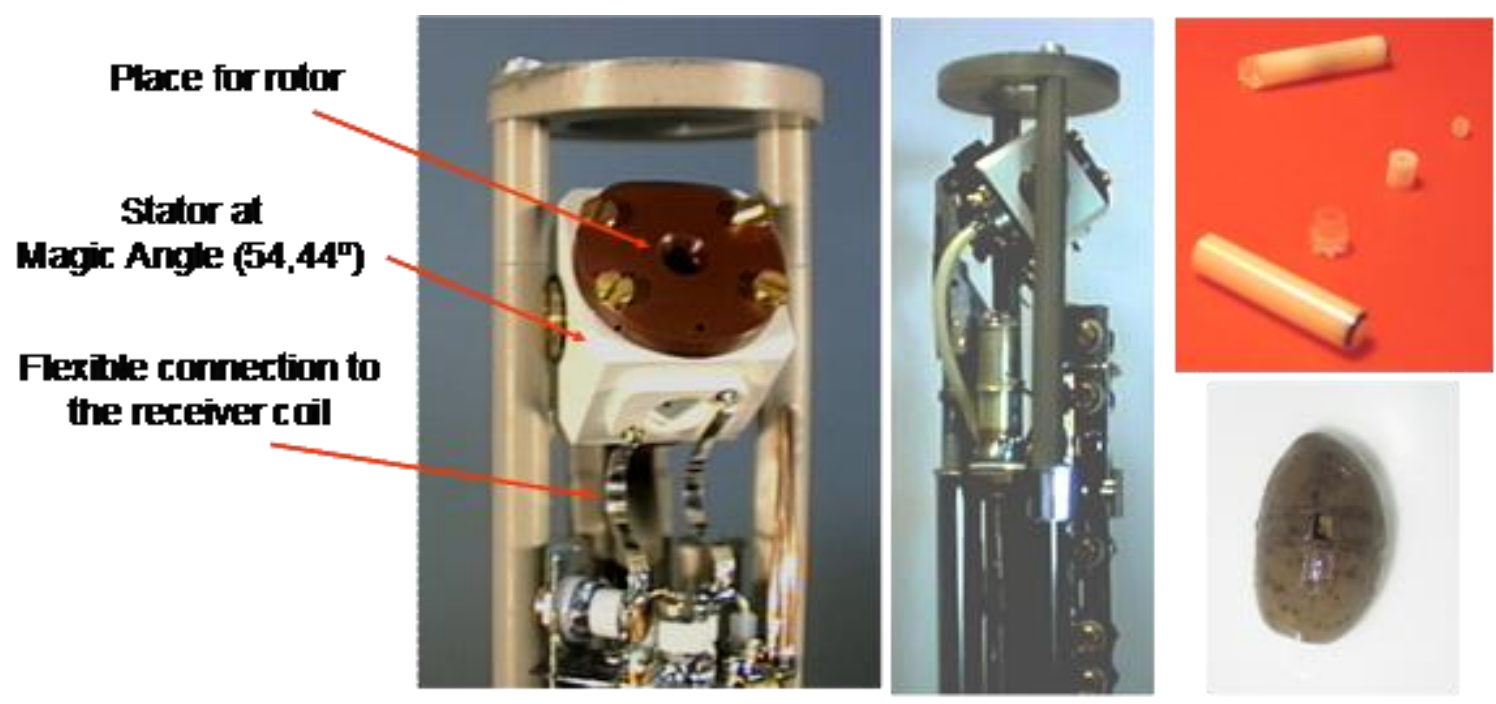

Fig.1. Instrumentation and sample. 


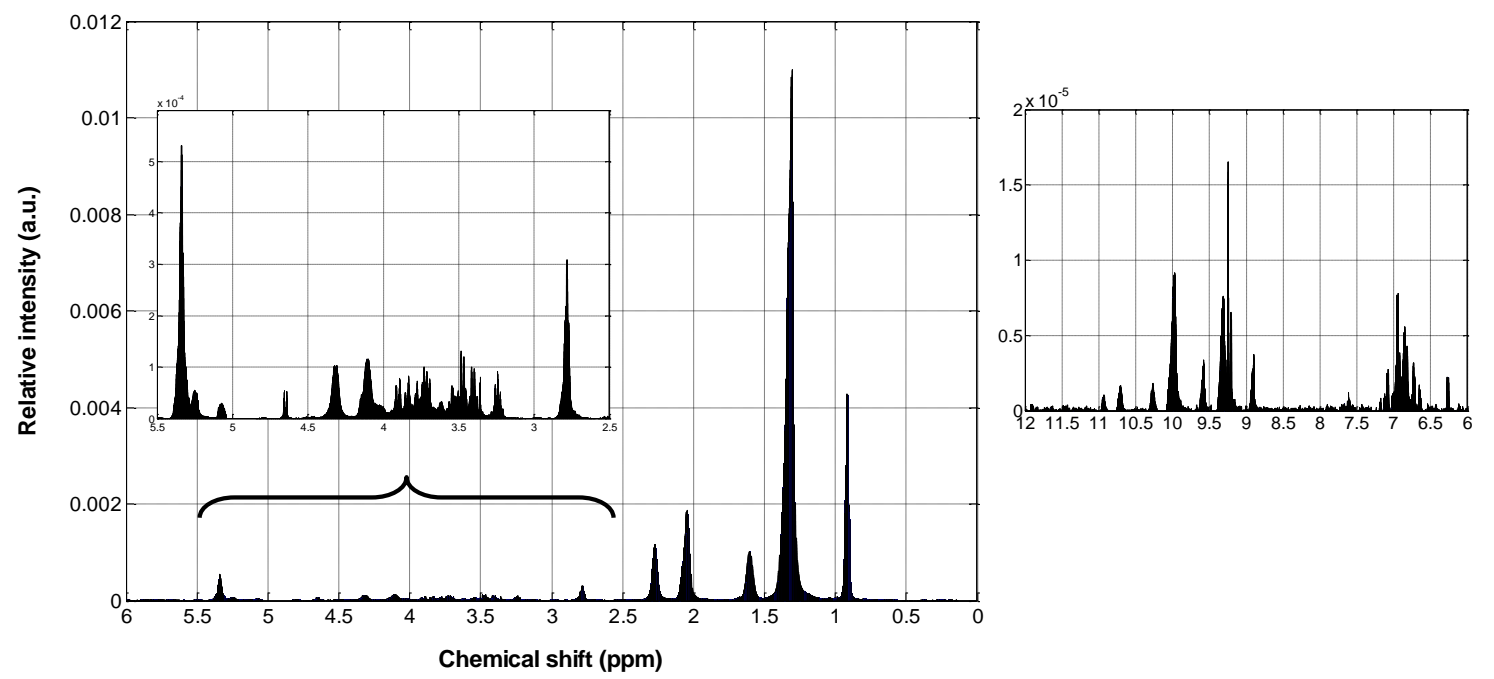

Fig. 2. Example of HR-NMR spectrum from a Picual sample.

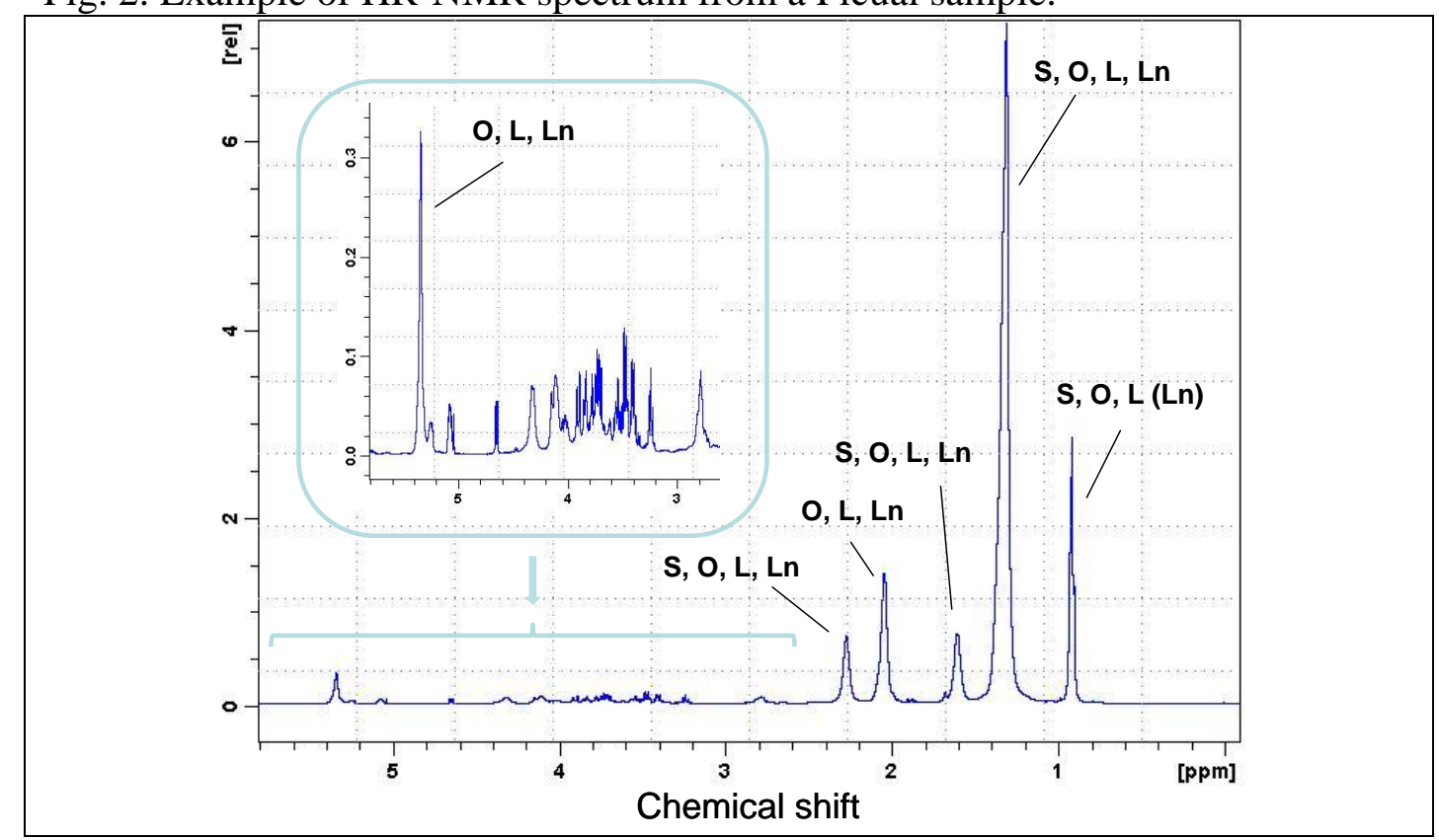




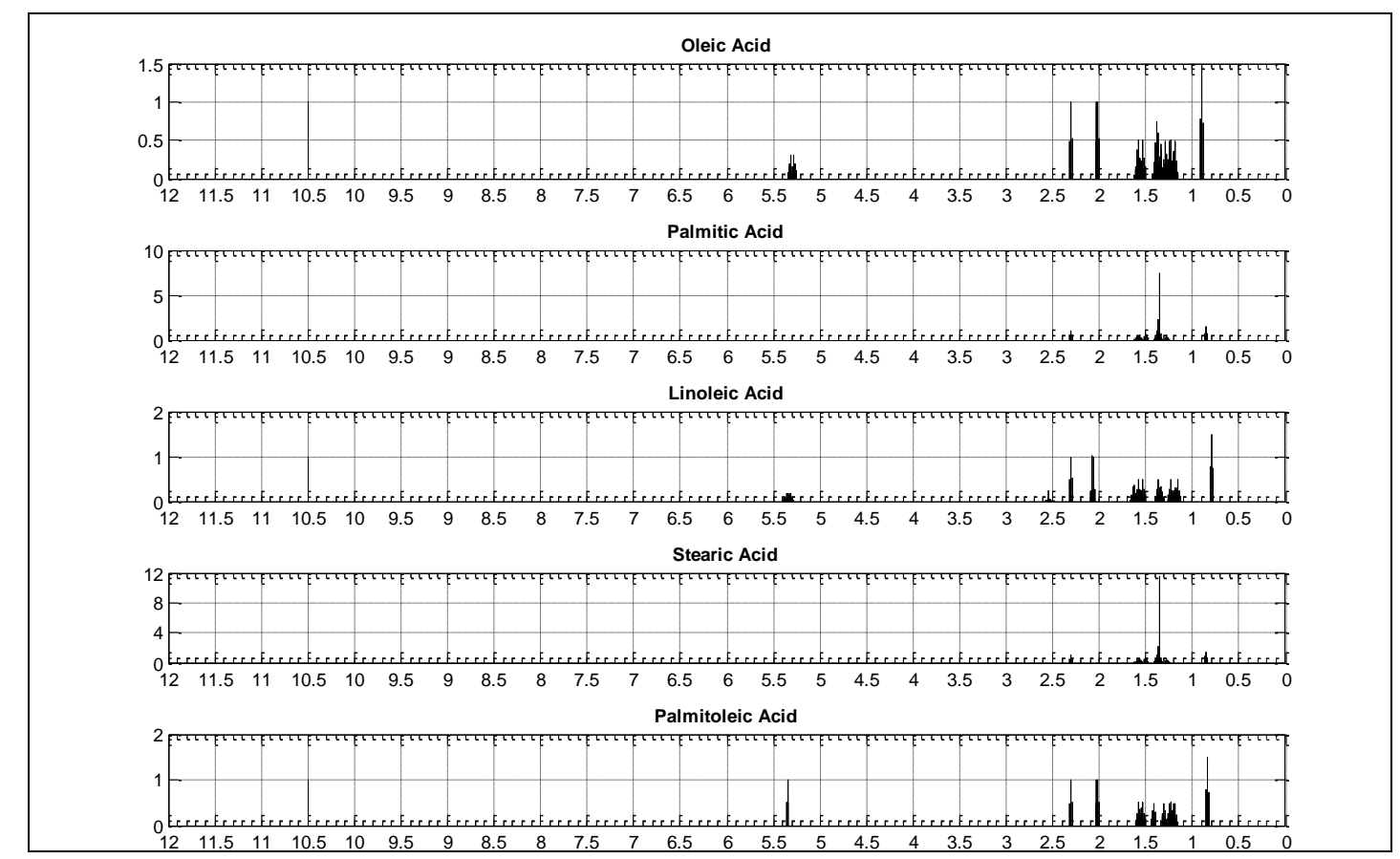

Fig. 3. Assignament of the main peaks to fatty acids (upper) according to simulated spectra (bottom).
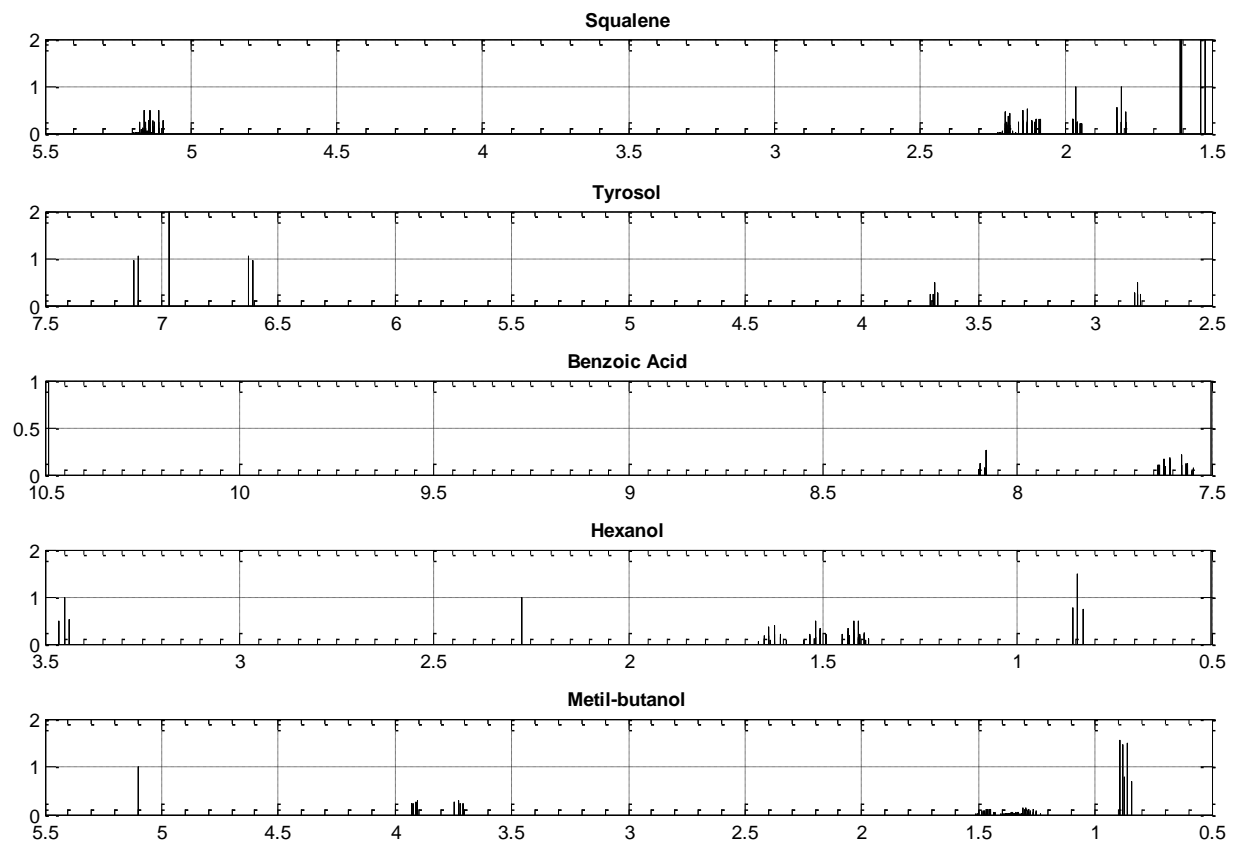

Fig. 4. Simulated spectra of minor components in olive. 


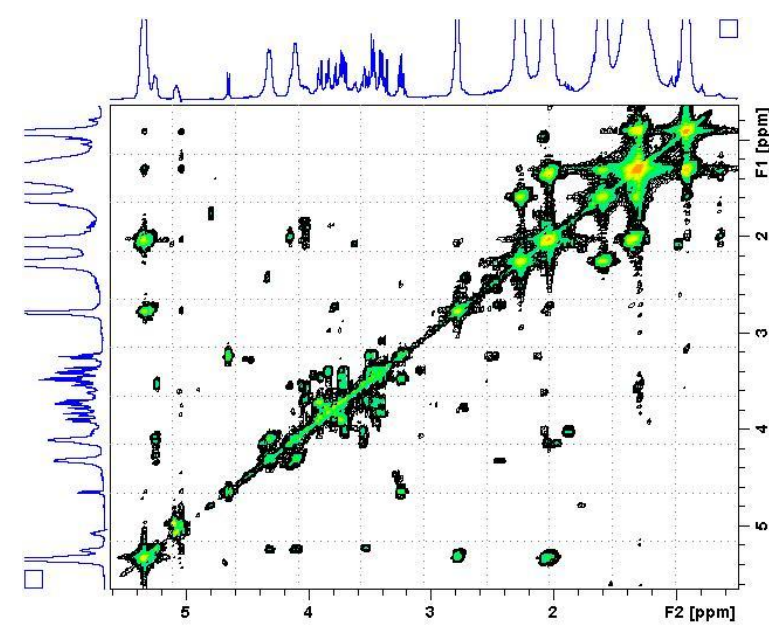

Fig. 5. Example of 2D HR-NMR spectrum (contour lines delimit the correlation between proton populations).

\section{$\underline{\text { Tables }}$}

Table 1. Summary of experiments.

\begin{tabular}{|c|c|c|c|}
\hline $\begin{array}{l}\text { Samples } \\
\text { (Num. fruits) }\end{array}$ & $\begin{array}{l}\text { NMR equipement } \\
\text { (Magnetic field strength } \\
\text { in Tesla) }\end{array}$ & NMR output & Objetive \\
\hline $\begin{array}{l}\text { Picual (4) } \\
\text { Arbequina (4) }\end{array}$ & \multirow[b]{2}{*}{ Bruker AMX (11.75 T) } & $\begin{array}{l}\text { 1D HR-NMR } \\
\text { spectra }\end{array}$ & $\begin{array}{l}\text { Spectroscopic } \\
\text { fingerprinting }\end{array}$ \\
\hline $\begin{array}{l}\text { Frantoio (4) } \\
\text { Descendants (20) } \\
{[\text { FxP, AxP, PxA] }}\end{array}$ & & $\begin{array}{l}\text { 2D HR-NMR } \\
\text { spectra }\end{array}$ & $\begin{array}{l}\text { Correlation between } \\
\text { peaks of the same } \\
\text { metabolite }\end{array}$ \\
\hline
\end{tabular}

Table 2. Spectral variables (chemical shifts in $\mathrm{ppm}$ ) with significant differences between olive groups acording to ANOVA. Normal frnt: 5\% significance; italic-bold font: $1 \%$ significance. A stands for Arbequina; P stands for Picual; F stands for Frantoio.

\begin{tabular}{lccccccc}
\hline Olive groups & \multicolumn{7}{c}{ Spectral variables (chemical shifts in ppm) } \\
\hline \multirow{2}{*}{ A vs P } & 0.92 & 1.61 & 3.24 & 3.40 & 3.53 & 3.69 & 3.73 \\
& 3.77 & 3.81 & 3.85 & 3.89 & 4.01 & 4.63 & 4.67 \\
& 5.35 & 6.86 & 7.10 & & & & \\
\hline \multirow{2}{*}{ A vs F } & 1.33 & 3.69 & 3.77 & 3.85 & 3.89 & 4.63 & 9.26 \\
& $\mathbf{9 . 3 4}$ & 9.58 & 9.99 & & & & \\
\hline P vs F & 6.65 & \multicolumn{7}{c}{} & & & & \\
\hline F vs FxP & 1.33 & $\mathbf{9 . 3 4}$ & 9.58 & 9.99 & & & \\
\hline P vs FxP & 1.61 & 7 & & & & \\
\hline
\end{tabular}


